\title{
Behaviour change intervention to reduce caregivers' exposure to patients' oral and nasal secretions in Bangladesh
}

\author{
Emily S Gurley, M Saiful Islam, Nazmun Nahar, Rebeca Sultana, M Jahangir Hossain, Nusrat Homaira, \\ Shahana Parveen, Tania Naushin, Mahbub-ul Alam, AKM Dawlat Khan, NM Rabiul A Chowdhury, \\ Dorothy Southern, Stephen P Luby \\ International Centre for Diarrhoeal Disease Research, Dhaka, Bangladesh
}

doi: 10.3396/ijic.v9i2.017.13

\begin{abstract}
Family caregivers are at risk for acquiring Nipah virus from patients in Bangladesh through exposure to patients' respiratory secretions. We conducted a formative study to assess the acceptability and feasibility of behaviour change messages to reduce this exposure in a hospital in Bangladesh. We delivered a bar of soap with behaviour change messages to caregivers asking them to: 1) wash hands with soap at key times; 2) not eat patient's leftover food; 3) sleep with their back to the patient or with the patient's face to their chest; and 4) maintain more than one hand's distance between patient's and caregiver's faces. Structured observations and semi-structured interviews with caregivers were used to assess acceptability and feasibility. Caregivers of 15 patients were enrolled. We observed an 172 opportunities for caregivers to wash their hands, and in 20 (12\%) of these opportunities caregivers washed hands with soap. Caregivers cited an inability to leave a severely ill patient unattended and lack of access to water as barriers to handwashing. Caregivers abstained from sharing food with patients in $82 \%$ (61/74) of observations with an opportunity to do so, and followed our sleeping suggestions in $88 \%(113 / 128)$. In only $12 \%(40 / 336)$ of observation sessions did the caregiver keep their face more than one hand's distance from the patient's. Behavioural messages regarding sleeping position and foodsharing were both acceptable and feasible; maintaining a distance from the patient was neither. Handwashing was acceptable, but not feasible. Interventions to target this at-risk group should include improved access to handwashing stations.
\end{abstract}

Key words

Nipah virus; Hand hygiene; Caregivers; Infection control; Bangladesh

\author{
Corresponding Author \\ Emily S Gurley \\ International Centre for Diarrhoeal Disease Research, Dhaka, Bangladesh \\ Email: egurley@icddrb.org
}




\section{Introduction}

Social norms in Bangladesh dictate that when someone becomes ill, they should receive close, attentive care from family and loved ones. ${ }^{1}$ As the severity of the disease increases, so does the perceived need for close emotional and physical support to the patient. ${ }^{1}$ This social expectation, coupled with limited human resources in Bangladeshi hospitals, means that family caregivers provide the majority of hands-on care in the community and the hospital setting. ${ }^{2}$

Many infectious diseases are spread through respiratory droplets, including emerging pathogens such as the coronavirus that has caused severe acute respiratory syndrome (SARS), Nipah virus, and new strains of influenza virus.-7 In Bangladesh, family caregivers often have intense exposure to patients' oral and nasal secretions during hospitalization. ${ }^{1,2,8}$ As a result, Nipah virus has been repeatedly transmitted to relatives or friends who cared for Nipah patients outbreaks in Bangladesh. ${ }^{6,8-11}$ During Nipah virus outbreaks in Bangladesh, family caregivers reported very close physical contact with patients, including sharing a bed. . $^{1,8,10,11}$

Bangladesh continues to experience almost yearly outbreaks of Nipah virus encephalitis, ${ }^{9,12}$ yet there are no demonstrably effective interventions to reduce risk of infection for family caregivers. Given the epidemiological evidence of transmission through respiratory droplets or hand contamination ${ }^{8,10}$ and repeated isolation of Nipah virus from patient respiratory specimens, ${ }^{13,14}$ effective interventions to prevent transmission in the hospital setting are needed.

Formative research for intervention development aims to identify the target group for the intervention and incorporate the perspective of this group to better inform the intervention design. ${ }^{15-18}$ Major components of such formative research include defining the population at risk, the behaviour associated with increased risk, as well as the feasibility and acceptability of the intervention. ${ }^{15}$ We had previously identified that caregivers were at risk for Nipah infection and epidemiological studies suggested that receiving a cough in the face from a Nipah patient put caregivers at risk for infection, but that handwahsing with soap was protective. ${ }^{8,10}$ Therefore, in this study we aimed to assess the acceptability and feasibility of behaviour change messages to reduce caregiver exposure to patient respiratory secretions.

\section{Methods}

Five researchers trained in qualitative methods from icddr,b (formerly known as the International Centre for Diarrheal Diseases Research, Bangladesh) delivered messages to family caregivers of patients with meningo-encephalitis at Rangpur Medical College Hospital, a tertiary care public teaching facility in northwestern Bangladesh with approximately 350 beds. The hospital, part of our meningo-encephalitis surveillance network, is typically crowded with patients being cared for on the floor when no beds are available. We assessed the acceptability and feasibility of the behaviour using structured observation and indepth interviews with caregivers.

\section{Proposed behaviour change messages}

The messages targeted the behaviour we believed posed the greatest risk for caregivers, based on our previous research: eating with contaminated hands, eating food contaminated by patient saliva, and receiving a cough in the face, either while sleeping next to or while comforting the patient. Handwashing with soap was the first behaviour promoted by our behaviour change messages. We counselled caregivers to wash their hands before eating, after feeding the patient, and after cleaning secretions from the patients' mouth and nose. In the second message we counselled caregivers to not eat patients' leftover food, and in the third, not to sleep face-to-face with the patient. We suggested alternate sleeping arrangements, including the caregiver sleeping on a different bed or the floor, and sleeping back to back, head to chest, or head to foot with the patient in the same bed. In the fourth message, we asked caregivers to comfort their patients by holding the patient's head to their chest, but to refrain from putting their face within one hand's width distance of the patient's face. We provided caregivers with a laminated $8 \times 10$ inch card depicting our suggested behaviour (Figure 1). In addition, we provided each caregiver with a bar of soap for handwashing since soap was not readily available at hospital handwahsing stations. 


\section{Assessing acceptability and feasibility of the messages}

We approached caregivers of 15 patients who were admitted with meningo-encephalitis during May and June 2009 and asked them to participate in the study. If the caregiver provided informed consent, we presented them with our behaviour change messages and gave them the laminated pictorial card and a bar of soap.

Research staff observed caregiving practices for the next five days at approximately 6-7 am, 12-2 pm, 5-6 pm, and 10-11 pm each day, until the patients were discharged from the hospital or died. Each 1-2 hour observation constituted one observation session. Timings of observations were chosen to maximize our ability to observe opportunities to perform the behaviour we promoted during eating and sleeping. As part of the consent process, participants were told that observers were collecting information about patient caregiving practices in their ward; however, the observers attempted to remain as inconspicuous as possible. Research staff were prohibited from observing behaviour in a ward where they had delivered behaviour change messages to caregivers so that observers were unknown to caregivers. Observers sat where they could see caregiving behaviour, but out of the line of sight of caregivers. Detailed notes were taken during each observation. At the end of every observation session, each of the four behaviour was given a compliance code indicating if an opportunity for the behaviour had been observed, and if so, if the behaviour had been performed.

Our behaviour messages recommended that caregivers wash their hands before eating and after cleaning secretions from the patient's mouth and nose, so we defined an opportunity to wash hands as any time a caregiver performed these behaviour during our observation. We then calculated the proportion of times that we observed handwashing with soap following one of these opportunities. For sharing patient food and utensils, we followed a similar process whereby we noted each time the caregiver ate food and calculated the proportion of times the food consumed was leftover patient food. Each time the caregiver was observed sleeping we noted their sleeping position in relation to the patient and counted the number of times that the caregiver adopted a sleeping position that we had recommended. Every observation session was regarded as an opportunity for the caregiver to keep their face $>1$ hand width's distance from the patient's face.

Caregivers were asked to participate in an exit interview prior to departure from the hospital or after 5 days of observation, whichever came first. Caregivers of patients who died were interviewed in their homes approximately two weeks after the death. The interviewer asked the caregiver open-ended questions about their recall of the behaviour messages, the acceptability of the messages, and probed about the barriers they faced in performing the suggested behaviour. The observation compliance codes were used as a tool for the interviewer to determine whether or not the caregiver was able to perform the behaviour. Interviewers probed specifically about behaviour the caregiver did not perform to better understand barriers they faced. Detailed notes were taken during the interview and the responses were summarized based on the major barriers in performing behaviour noted by respondents.

\section{Human subjects considerations}

All participants provided informed, written consent for participation in the study. This protocol was reviewed and approved by icddr,b's ethical review board. Patients and caregivers photographed performing the behaviour for the pictorial card provided signed consents for the use of these photos for public dissemination.

\section{Results}

We enrolled 17 caregivers of 15 patients in our study. Six of the patients were children and 9 were adults aged $\geq 18$ years (Table I). Thirteen caregivers were women (87\%) and 10 patients were cared for by their mothers (Table I). We conducted 336 observation sessions with these caregivers, accounting for 430 total hours of caregiver behaviour observation. We completed exit interviews with all 17 caregivers; two patients each had two caregivers who received the behaviour change messages and the caregivers were interviewed together upon exit from the study. One patient died in the hospital and the interview was completed at home two weeks after the death.

Although caregivers frequently rinsed hands with stored water at the bedside during key times, they only 
Table I. Demographic characteristics of and relationship between patients and their caregivers enrolled in this study

\begin{tabular}{c|cc|cc|l} 
Number & \multicolumn{2}{c}{ Patient } & \multicolumn{2}{c}{ Caregiver } & Relationship of caregiver to patient \\
\hline & Age & Sex & Age & Sex & \\
\hline 1 & 37 & F & 58 & F & Mother \\
\hline 2 & 1.8 & M & 20 & F & Mother \\
\hline $3^{*}$ & 25 & M & 20 & F & Wife \\
\hline & & & 48 & M & Father \\
\hline 4 & 19 & M & 50 & F & Mother \\
\hline 5 & 6 & F & 28 & F & Mother \\
\hline 6 & 18 & M & 45 & F & Mother \\
\hline 7 & 28 & F & 33 & M & Husband \\
\hline 8 & 1.3 & M & 25 & F & Mother \\
\hline 9 & 6 & F & 25 & F & Mother \\
\hline 10 & 22 & F & 25 & F & Sister \\
\hline 11 & 45 & F & 50 & M & Husband \\
\hline 12 & 6 & M & 35 & F & Mother \\
\hline 13 & 48 & F & 22 & F & Daughter \\
\hline $14^{*}$ & 15 & M & 35 & F & Aunt \\
\hline & & & 42 & M & Father \\
\hline 15 & 18 & F & 50 & F & Mother \\
\hline
\end{tabular}

*Patients 3 and 14 had two caregivers

washed hands with soap in $12 \%$ (20/172) of observed opportunities to do so. In $82 \%$ (61/74) of observations where caregivers fed patients we observed that patient food was not consumed by caregivers and that they did not share plates, glasses or utensils. In 88\% (113/128) of observation sessions when the caregiver was sleeping, he/she was not sleeping face-to-face with the patient as suggested by our messages. During 11 observations (9\%) of caregiver sleeping, the caregiver was sleeping head to toe with the patient. Caregivers kept their face more than a hand's width distance from their patient's face in $12 \%$ of $(40 / 336)$ observations.

All caregivers remembered our messages during exit interviews and they over-reported practicing the behaviour we promoted compared to our observation notes. All but one caregiver reported that they faced barriers to performing some of the behaviour.

During exit interviews caregivers described numerous barriers to handwashing with soap (Table II). There were two locations where caregivers could access running water: a bathroom located on each ward with intermittent water supply, and a tubewell pump located on the ground floor of the hospital which was two floors down from the ward. Water from the tubewell was considered safer for drinking, so most caregivers filled plastic containers with tubewell water and stored it by their beds. This stored tubewell water was precious because both caregivers and patients used it for drinking, and because time and containers required to fetch the water were both in short supply. As one male caregiver told us:

"Stored water is like calculated water. I had to be very careful about using stored water. Say, if I give water to my patient to drink, then I may not have enough water to wash my hands as you suggested. If I wash my hands frequently, than I may not get time to go outside again to collect water. I will face scarcity of water while feeding medicine to my patient. If I had water available 
Table II. Barriers to performing intervention behaviour mentioned by caregivers $(\mathrm{N}=15)$

\section{Barriers to handwashing with soap}

- Forgot about message due to concern and work associated with patient care

- Inability to leave patient unattended

- Unable to collect sufficient water from tubewell

- Limited availability of water in the bathroom

- Long lines to access water in bathrooms

- Caregiver reluctance to enter dirty bathrooms for handwashing

\section{Reasons for sharing food or plates and utensils with patient}

- Only brought 1 set of dishes or utensils from home

\section{Barriers to sleeping back to back, in separate beds, or face to chest}

- Patient demands to sleep face to face with mother

- Multiple caregivers or patients sharing a single bed

\section{Barriers to keeping face more than one hand width's distance from patient's face}

- Caregiver trying to hear patient words/ patient speaking softly

- Patient desire for close contact

- Caregiver desire for close contact

- had to be close to feed them through the NG tube

- Difficult to maintain when carrying or moving the patient or holding during convulsions

" + " = mentioned by 1-6 caregivers

"++" = mentioned by 7 or more caregivers

to me, within my reach, it would be easier for me to maintain your suggestions."

Caregivers complained that bathrooms were dirty with urine and faeces, lacked water during most of the day, and were crowded when water was available. These circumstances greatly reduced their access to water and opportunities to wash hands with soap. One caregiver explained his experiences with accessing water:

"Many times I heard from other caregivers that the water supply is available in the bathroom. Then after taking care of my patient, I went to the bathroom with soap for handwashing but I could not wash my hands because in the meantime, the water supply had stopped. I came back (to the bed) and rinsed my hands only with a little amount of stored water. After that, I was confused and scared because I washed hands but without soap so the germs might not have been removed from my hands."

The logistical barrier to handwashing with soap most frequently mentioned was the inability of the caregiver to leave the patient alone. Caregivers sometimes reported that patients would strongly object if the caregiver left their side. Patients in this study were frequently severely ill and many experienced convulsions and required constant attention. As one caregiver told us:

"If I went to wash my hands with soap, my patient would be alone on the ward. But it was not possible to leave him alone due to his convulsions. I had to hold him tightly during his convulsions. .. So, I couldn't leave my patient to wash my hands with soap."

Caregivers were under tremendous stress because of the seriousness of their patient's illness and the numerous caregiving tasks including feeding the patient, arranging money to pay for services and medicine, providing emotional support to the patient, and concern about the responsibilities they had left at home. As one mother told us,

"Is it possible to maintain any suggestions regarding care giving practices for a mother when her child's condition is not good? How is it possible to wash my hands carefully in this situation?" 
Despite these limitations, we did see caregivers wash their hands with soap 20 times. There were two scenarios when we observed handwashing with soap: either in the context of washing clothes or plates with soap, or when caregiver hands were visibly soiled with patient excretions or oil after providing the patient with therapeutic massage. As one caregiver told us, "After cleaning that (the urinary catheter) I washed my hands with soap because all of us dislike touching urine. Everyone will wash their hands after that."

The hospital provided food for patients three times a day. Sometimes, caregivers also consumed this food and at other times food was brought from outside the hospital or caregivers left the hospital to eat. This behaviour was made easier by the fact that many patients were unable to eat solid food due to their illness. One barrier to performing this behaviour was that caregivers frequently only brought one plate, drinking glass and set of utensils from home (Table II)

Caregivers were only rarely observed sleeping face-toface with patients. Although, one barrier to performing this behaviour was insufficient sleeping space when two paediatric patients and their caregivers shared a single bed (Table II). When possible, caregivers slept on the floor or in another bed. However, they usually just slept head-to-foot with the patient, or with the patient's head at their chest, particularly in the case of child patients. Caregivers reported that the smell of patients' breath was unpleasant and that this served as a cue to change sleeping position.

Caregivers faced two main barriers in keeping a distance from the patient's face. Due to severity of illness, many patients were unable to speak loudly. Therefore, caregivers reported that they had to bring their face close to the patient's to hear their whispered requests. Emotional desire for closeness, either on the part of the caregiver or the patient, also resulted in close contact between faces (Table II).

\section{Discussion}

Based on our observations and interviews with caregivers, some of the behaviour we promoted to reduce caregiver exposure to patient oral and nasal secretions were more feasible and acceptable than others. Specifically, our study suggests that refraining from sharing food and utensils and not sleeping face- to-face with patients were acceptable and practical behaviour. Interventions to prevent person-to-person transmission of Nipah virus from patients to caregivers should include behaviour change messages about sharing food and sleeping arrangements. However, handwashing with soap and maintaining a distance between patient's and caregiver's faces were much less frequently observed. Caregivers reported that handwashing was an acceptable practice, but not feasible because of poor access to a handwashing station and the inability to leave severely ill patients unattended. Given the epidemiologic data suggesting that handwashing with soap was protective for Nipah infection $^{8}$ and the willingness of caregivers to wash hands, future interventions in the hospital setting should include strategies to increase access to handwashing facilities. Caregivers frequently communicated with and comforted patients by bringing their faces close together. Keeping a physical distance from patients was less acceptable and feasible for caregivers because of the need to communicate with patients. Findings from our study suggest that risk communication messages are unlikely to substantially reduce this contact. However, given the high risk associated with close contact with Nipah patients, ${ }^{8}$ even modest reductions in this close contact may confer some protection to caregivers. Caregiver use of medical masks to provide barrier protection is another possible option for reducing this risk but we did not investigate the feasibility and acceptability of mask use in this study because medical masks are not routinely available in hospital settings in Bangladesh. The use of medical masks to prevent contact with patient respiratory secretions should be explored in future studies. Longer-term strategies to provide professional nursing care and isolate these potentially infectious patients would be most effective but would require substantial investments in infrastructure and personnel.

Hand contamination is a well described risk for infection with numerous viral pathogens ${ }^{4,19-21}$ and caregivers in this setting remain at significant risk from contaminated hands. Some studies have suggested that increased access to hand hygiene infrastructure is associated with increased hand cleaning, both in the hospital and community setting. ${ }^{22-24}$ Indeed, major barriers to handwashing with soap in this study were an inability to leave the patient unattended to access a handwashing station or to remember to wash 


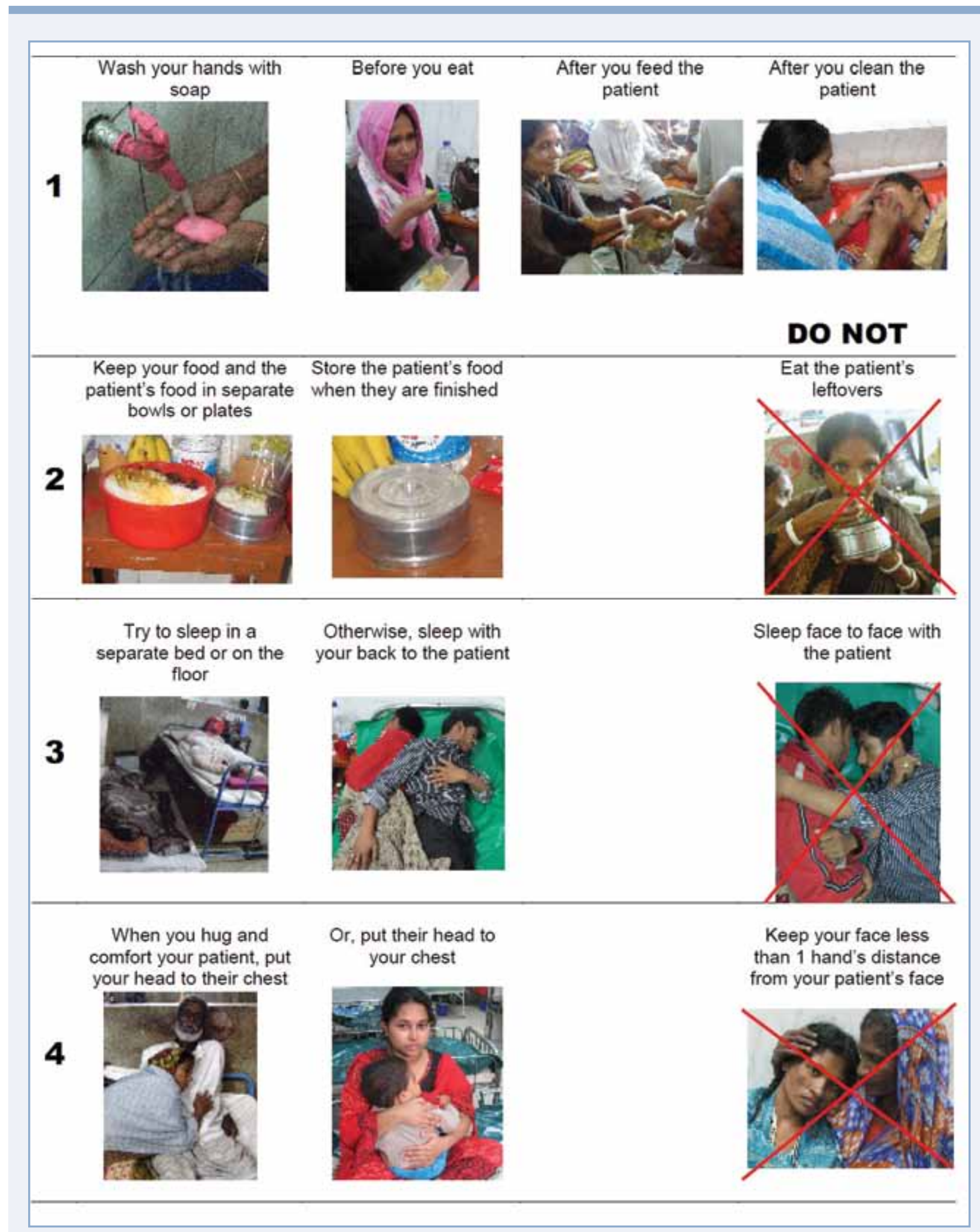

Figure 1. Laminated card given to study participants which depicts both the caregiving behaviour we promoted and those we discouraged 
hands during a stressful time. Provision of water for handwashing at the bedside could improve access as well as provide a visual cue to action. Investments in installation of sinks throughout the ward could improve access to a handwashing station. Water storage tanks could be built to provide access to water in bathrooms and handwashing stations even when the municipal supply water is turned off. In addition, a short-term strategy could be to provide buckets with spigots for storage and use of water for drinking and handwashing on the ward. Caregivers already bring tubewell water to the bedside in small containers for drinking and rinsing hands. Perhaps if larger vessels for carrying and dispensing water were provided caregivers would be willing to bring larger amounts of tubewell water to the bedside. Additional plastic buckets located under the spigot could catch waste water for disposal. Hand sanitizers are another option to consider in this setting for cleaning hands, particularly given the intermittent water supply. Although local sanitizers are more costly than soap (US\$.50 per bottle compared to US\$.20 per bar), cheaper alternative hand sanitizers could be made at the hospital using the $\mathrm{WHO}$ recipe. ${ }^{25}$ Disadvantages of the hand sanitizer strategy are that hand sanitizers are currently foreign to caregivers in this setting and mobilizing hospital staff to maintain a constant supply of hand sanitizers could be difficult. Additional research on the feasibility and acceptability of these hand hygiene strategies could be used to inform future interventions.

This formative research was conducted among only a small number of caregivers in a government hospital so it may not be representative of all persons caring for patients with Nipah or meningo-encephalitis in Bangladesh. However, responses from our study participants were highly consistent and are likely applicable to caregivers of a seriously ill patients in this and similar settings. The behavioural messages were more acceptable and feasible based on caregiver interview data compared to our observations and this could be partially accounted for by courtesy bias. This highlights the need for observations in assessing acceptability and feasibility of behaviour. ${ }^{26,27}$

Family caregivers are an integral part of the healthcare system in Bangladesh ${ }^{1,2}$ and efforts to reduce their risk of disease deserve continued attention. These caregivers have close and sustained contact with infectious patients in the hospital setting and if infected, could pose a risk to their broader communities of residence. Findings from this study suggest that family caregivers are interested in preventing infections and that some behaviour to reduce their exposure to patient secretions are feasible and acceptable. Importantly, however, handwashing with soap appeared to be acceptable but not feasible due to logistical constraints. Practical behaviour change interventions to reduce family caregiver risk of infection should be developed and tested to determine if they are effective in reducing caregiver exposure to potentially infectious secretions and body fluids. Once effective interventions are identified, they should be formally incorporated into hospital infection control initiatives and outbreak response activities.

\section{Acknowledgements}

This study was conducted with support from the Centers for Disease Control and Prevention, USA (CDC), cooperative agreement U01/C1000298. icddr,b acknowledges CDC's support to its research efforts.

The authors wish to thank Rangpur Medical College Hospital administrators and healthcare professionals for their willingness to participate in the study and the caregivers who gave us their time, attention, and invaluable feedback on our behaviour messages.

All authors confirm that they have no conflicts of interest.

\section{References}

1. Blum LS, Khan R, Nahar N, Breiman RF. In-depth assessment of an outbreak of Nipah encephalitis with person-to-person transmission in Bangladesh: implications for prevention and control strategies. The American Journal of Tropical Medicine and Hygiene 2009; 80(1): 96-102. http://intl.ajtmh.org/ content/80/1/96.full

2. Hadley MB, Blum LS, Mujaddid S, et al. Why Bangladeshi nurses avoid 'nursing': social and structural factors on hospital wards in Bangladesh. Social Science \& Medicine 2007; 64(6): 11661177. http://dx.doi.org/10.1016/j.socscimed.2006.06.030

3. Chen WQ, Ling WH, Lu CY, et al. Which preventive measures might protect health care workers from SARS? BMC Public Health 2009; 9: 81. http://dx.doi.org/10.1186/1471-2458-981

4. Jefferson T, Del Mar C, Dooley L, et al. Physical interventions to interrupt or reduce the spread of respiratory viruses: systematic review. British Medical Journal 2009; 339: b3675. http://dx.doi.org/10.1136/bmj.b3675 
5. Johnson DF, Druce JD, Birch C, Grayson ML. A quantitative assessment of the efficacy of surgical and N95 masks to filter influenza virus in patients with acute influenza infection. Clinical Infectious Diseases 2009; 49(2): 275-277. http:// dx.doi.org/10.1086/600041

6. Luby SP, Gurley ES, Hossain MJ. Transmission of human infection with Nipah virus. Clinical Infectious Diseases 2009; 49(11): 1743-1748. http://dx.doi.org/10.1086/647951

7. Mubareka S, Lowen AC, Steel J, Coates AL, Garcia-Sastre A, Palese P. Transmission of influenza virus via aerosols and fomites in the guinea pig model. The Journal of Infectious Diseases 2009; 199(6): 858-865. http://dx.doi.org/10.1086/597073

8. Gurley ES, Montgomery JM, Hossain MJ, et al. Person-to-person transmission of Nipah virus in a Bangladeshi community. Emerging Infectious Diseases 2007; 13(7): 1031-1037. http:// dx.doi.org/10.3201/eid1307.061128

9. ICDDRB. Nipah outbreak in Faridpur District, Bangladesh 2010. Health and Science Bulletin 2010; 8(2): 6-11. (http:// www.icddrb.org/what-we-do/publications/cat_view/52publications/10042-icddrb-periodicals/10048-healthand-science-bulletin-bangla-and-english/10102-vol-8no-2-english-2010/11757-nipah-outbreak-in-faridpurdistrictbangladesh-2010

10. Homaira N, Rahman M, Hossain MJ, et al. Nipah virus outbreak with person-to-person transmission in a district of Bangladesh, 2007. Epidemiology and Infection 2010; 138(11): 1630-1636. http://dx.doi.org/10.1017/S0950268810000695

11. Homaira N, Rahman M, Hossain MJ, et al. Cluster of Nipah virus infection, Kushtia District, Bangladesh, 2007. PLoS ONE 2010; 5(10): e13570. http://dx.doi.org/10.1371/journal. pone.0013570

12. Luby SP, Hossain MJ, Gurley ES, et al. Recurrent zoonotic transmission of Nipah virus into humans, Bangladesh, 20012007. Emerging Infectious Diseases 2009; 15(8): 1229-1235. http://dx.doi.org/10.3201/eid1508.081237

13. Chua KB, Lam SK, Goh KJ, et al. The presence of Nipah virus in respiratory secretions and urine of patients during an outbreak of Nipah virus encephalitis in Malaysia. The Journal of Infection 2001; 42(1): 40-43. http://dx.doi.org/10.1053/jinf.2000.0782

14. Harcourt BH, Lowe L, Tamin A, et al. Genetic characterization of Nipah virus, Bangladesh, 2004. Emerging Infectious Diseases 2005; 11(10): 1594-1597. http://dx.doi.org/10.3201/ eid1110.050513

15. Curtis V, Kanki B, Cousens S, Sanou A, Diallo I, Mertens T. Dirt and diarrhoea: formative research in hygiene promotion programmes. Health Policy Plan 1997; 12(2): 122-131. http:// dx.doi.org/10.1093/heapol/12.2.122

16. Gittelsohn J, Steckler A, Johnson CC, et al. Formative research in school and community-based health programs and studies: "state of the art" and the TAAG approach. Health Educ Behav 2006; 33(1): 25-39. http://dx.doi. org/10.1177/1090198105282412
17. Higgins DL, O'Reilly K, Tashima N, et al. Using formative research to lay the foundation for community level HIV prevention efforts: an example from the AIDS Community Demonstration Projects. Public Health Rep 1996; 111(Suppl 1): 28-35. http://pubmedcentralcanada.ca/pmcc/articles/ PMC1382040/pdf/pubhealthrep00044-0030.pdf

18. Young DR, Johnson CC, Steckler A, et al. Data to action: using formative research to develop intervention programs to increase physical activity in adolescent girls. Health Educ Behav 2006; 33(1): 97-111. http://dx.doi.org/10.1177/1090198105282444

19. Ansari SA, Springthorpe VS, Sattar SA, Rivard S, Rahman $M$. Potential role of hands in the spread of respiratory viral infections: studies with human parainfluenza virus 3 and rhinovirus 14. Journal of Clinical Microbiology 1991; 29(10): 2115-2119. http://www.ncbi.nlm.nih.gov/pmc/articles/ PMC270283/

20. Jefferson T, Foxlee R, Del Mar C, et al. Interventions for the interruption or reduction of the spread of respiratory viruses. Cochrane Database of Systematic Reviews 2007; (4): CD006207. http://www.thecochranelibrary.com/userfiles/ ccoch/file/CD006207.pdf

21. Sandora TJ, Taveras EM, Shih MC, et al. A randomized, controlled trial of a multifaceted intervention including alcohol-based hand sanitizer and hand-hygiene education to reduce illness transmission in the home. Pediatrics 2005; 116(3): 587-594. http://dx.doi.org/10.1542/peds.2005-0199

22. Graham M. Frequency and duration of handwashing in an intensive care unit. American Journal of Infection Control 1990; 18(2): 77-81. http://dx.doi.org/10.1016/0196-6553(90)900857

23. Kaplan LM, McGuckin M. Increasing handwashing compliance with more accessible sinks. Infection Control 1986; 7(8): 408410. http://www.jstor.org/stable/30143990

24. Luby SP, Halder AK, Tronchet C, Akhter S, Bhuiya A, Johnston RB. Household characteristics associated with handwashing with soap in rural Bangladesh. The American Journal of Tropical Medicine and Hygiene 2009; 81(5): 882-887. http:// dx.doi.org/10.4269/ajtmh.2009.09-0031

25. WHO Guidelines on hand hygiene in healthcare, 2009. http:// whqlibdoc.who.int/publications/2009/9789241597906_eng. pdf

26. Halder AK, Tronchet C, Akhter S, Bhuiya A, Johnston R, Luby SP. Observed hand cleanliness and other measures of handwashing behavior in rural Bangladesh. BMC Public Health 2010; 10: 545. http://dx.doi.org/10.1186/1471-245810-545

27. Manun'Ebo M, Cousens S, Haggerty P, Kalengaie M, Ashworth A, Kirkwood B. Measuring hygiene practices: a comparison of questionnaires with direct observations in rural Zaire. Tropical Medicine and International Health 1997; 2(11): 1015-1021. http://dx.doi.org/10.1046/j.1365-3156.1997.d01-180.x 